\title{
Cryogenic linear Paul trap for cold highly charged ion experiments
}

M. Schwarz, O. O. Versolato, A. Windberger, F. R. Brunner, T. Ballance, S. N. Eberle, J. Ullrich, P. O. Schmidt, A. K. Hansen, A. D. Gingell, M. Drewsen, and J. R. Crespo López-Urrutia

Citation: Review of Scientific Instruments 83, 083115 (2012); doi: 10.1063/1.4742770

View online: https://doi.org/10.1063/1.4742770

View Table of Contents: http://aip.scitation.org/toc/rsi/83/8

Published by the American Institute of Physics

\section{Articles you may be interested in}

Cryogenic linear ion trap for accurate spectroscopy

Review of Scientific Instruments 67, 129 (1996); 10.1063/1.1146560

Cryogenic setup for trapped ion quantum computing

Review of Scientific Instruments 87, 113103 (2016); 10.1063/1.4966970

Minimization of ion micromotion in a Paul trap

Journal of Applied Physics 83, 5025 (1998); 10.1063/1.367318

Deceleration, precooling, and multi-pass stopping of highly charged ions in $\mathrm{Be}^{+}$Coulomb crystals

Review of Scientific Instruments 86, 103111 (2015); 10.1063/1.4934245

Coulomb crystals in a cryogenic Paul trap for sympathetic cooling of molecular ions and highly charged ions AIP Conference Proceedings 1521, 250 (2013); 10.1063/1.4796081

Compact radio-frequency resonator for cryogenic ion traps

Review of Scientific Instruments 83, 084705 (2012); 10.1063/1.4737889

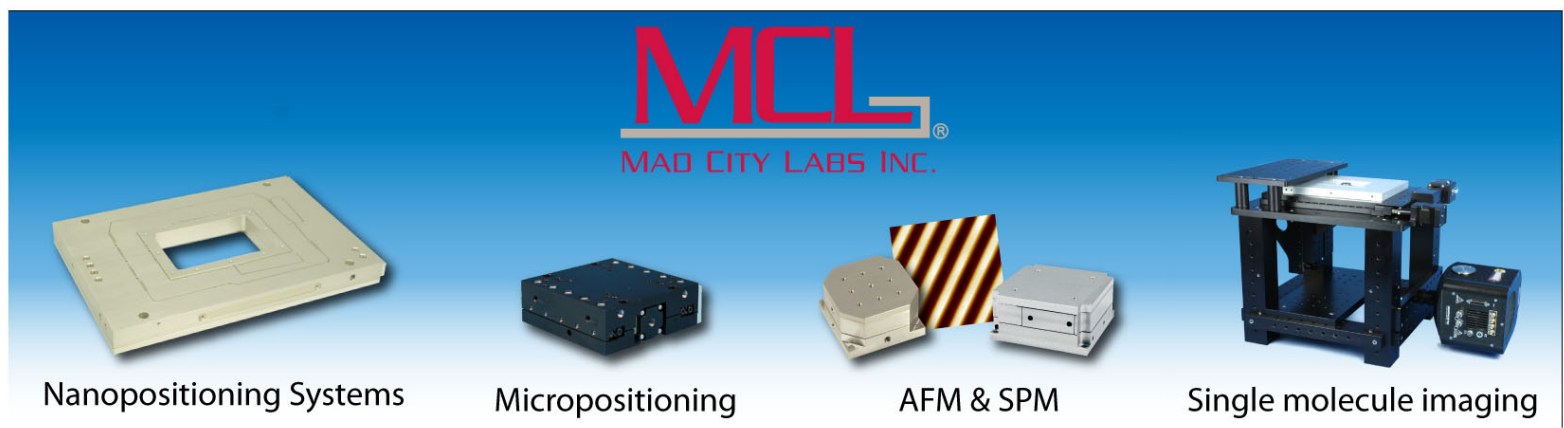




\title{
Cryogenic linear Paul trap for cold highly charged ion experiments
}

\author{
M. Schwarz, ${ }^{1, \text { a) }}$ O. O. Versolato, ${ }^{1,2}$ A. Windberger, ${ }^{1}$ F. R. Brunner, ${ }^{1}$ T. Ballance,${ }^{1}$ \\ S. N. Eberle, ${ }^{1}$ J. Ullrich, ${ }^{1,3}$ P. O. Schmidt, ${ }^{3,4}$ A. K. Hansen, ${ }^{5}$ A. D. Gingell, ${ }^{5}$ M. Drewsen, ${ }^{5}$ \\ and J. R. Crespo López-Urrutia ${ }^{1}$ \\ ${ }^{1}$ Max-Planck-Institut für Kernphysik, Saupfercheckweg 1, 69117 Heidelberg, Germany \\ ${ }^{2}$ University of Groningen, Kernfysisch Versneller Instituut, NL-9747 AA Groningen, The Netherlands \\ ${ }^{3}$ Physikalisch-Technische Bundesanstalt, Bundesallee 100, 38116 Braunschweig, Germany \\ ${ }^{4}$ Institut für Quantenoptik, Leibniz Universität Hannover, Welfengarten 1, 30167 Hannover, Germany \\ ${ }^{5}$ Department of Physics and Astronomy, University of Aarhus, DK-8000 Aarhus C, Denmark
}

(Received 28 March 2012; accepted 11 July 2012; published online 27 August 2012)

\begin{abstract}
Storage and cooling of highly charged ions require ultra-high vacuum levels obtainable by means of cryogenic methods. We have developed a linear Paul trap operating at $4 \mathrm{~K}$ capable of very long ion storage times of about $30 \mathrm{~h}$. A conservative upper bound of the $\mathrm{H}_{2}$ partial pressure of about $10^{-15} \mathrm{mbar}$ (at $4 \mathrm{~K}$ ) is obtained from this. External ion injection is possible and optimized optical access for lasers is provided, while exposure to black body radiation is minimized. First results of its operation with atomic and molecular ions are presented. An all-solid state laser system at $313 \mathrm{~nm}$ has been set up to provide cold $\mathrm{Be}^{+}$ions for sympathetic cooling of highly charged ions. (C) 2012 American Institute of Physics. [http://dx.doi.org/10.1063/1.4742770]
\end{abstract}

\section{INTRODUCTION}

Paul traps ${ }^{1-5}$ have found widespread applications in atomic and molecular physics, ${ }^{6,7}$ quantum computing, ${ }^{8-10}$ and frequency metrology. ${ }^{11,12}$ With the long ion storage times that are reached in them in combination with laser cooling, ${ }^{13,14}$ they are ideal tools for research with small particle numbers at very low translational temperatures. ${ }^{15-20}$ High-resolution laser spectroscopy benefits from these advantages, and thus currently the most accurate optical frequency measurements and comparisons are based on such traps. ${ }^{12,21}$ By applying sympathetic cooling ${ }^{22-25}$ via a co-trapped cooling ion, and with the recent introduction of quantum logic based detection schemes, ${ }^{26,27}$ it has become possible to detect the internal state of the spectroscopy ion after probing without resorting to a fast cycling transition in the spectroscopy ion. Using a series of laser pulses, entangling the internal states with a common motional mode, the internal state is mapped onto the cooling ion where it can be detected via the electron shelving technique $^{28}$ using a state-selective cycling transition. Other proposed schemes include the observation of coherent motion for the detection of the trapped ion excitation. ${ }^{27}$ Paul traps are the instruments of choice in laboratory searches for possible changes of fundamental constants, since they enable experiments with the low systematic uncertainties required to detect these tiny possible variations. ${ }^{12}$ Highly charged ions (HCIs) are particularly suited for such studies ${ }^{29,30}$ with the highest sensitivities predicted for atomic systems so far. HCIs are also sensitive probes of quantum electrodynamics (QED) ${ }^{31-34}$ and parity violating effects, ${ }^{35-37}$ and could also serve as optical frequency standards. ${ }^{38}$ Other applications of Paul traps include the investigation of cavity $\mathrm{QED}^{39-45}$ and cold molecular ions. ${ }^{18-20,46,47}$

Some of the open challenges in the field of ion trapping are the cooling of HCIs to temperatures necessary to

a)Electronic mail: m.schwarz@mpi-hd.mpg.de. high accuracy laser spectroscopy, ${ }^{33,48}$ for which schemes using Paul traps have been proposed, ${ }^{49}$ and the preparation of cold molecular ensembles in the rovibrational ground state. Both goals call for a large reduction of black body radiation and improvement of the vacuum conditions to well below $10^{-12}$ mbar to reduce collision-induced heating and ion loss by charge exchange. ${ }^{50}$

For these purposes, we have designed and built cryogenic Paul trap experiment (CryPTEx), a cryogenically cooled linear Paul trap, at the Max-Planck-Institut für Kernphysik (MPIK) in Heidelberg in collaboration with the Ion Trap Group at Aarhus University (AU). This trap has multiple optical access points, provides connectivity to external ion beam sources, and exhibits excellent mechanical, electrical, and thermal stability. The cryogenic shielding of the inner sections overcomes the above-mentioned limitations of room-temperature traps. The advantages of a cryogenic environment ${ }^{51}$ facilitate the production of ultra-high vacuum (UHV). In this paper, we describe the setup of the apparatus and its performance, as well as initial experiments using ion beams and laser cooled atomic and molecular ion crystals. There have been earlier cryogenic designs, such as that of Poitzsch ${ }^{52}$ and others. ${ }^{53,54}$ The main difference between those and the present apparatus is the usage of a cryocooler, ${ }^{55}$ the enhanced optical access, and the ion injection capability that is indispensable for HCI related work.

\section{SPECIFICATIONS}

The requirements for the system design were dictated by its intended application: operation of the trap at $4 \mathrm{~K}$ temperature (for vacuum purposes as well as to reduce black body radiation) with a thermal load of less than $1 \mathrm{~W}$; capability to utilize radiofrequency (rf) voltages as high as $1000 \mathrm{~V}$ peakto-peak in the 1-10 MHz range; access to the trap center by 15 optical ports; provision of an achromatic ion fluorescence 


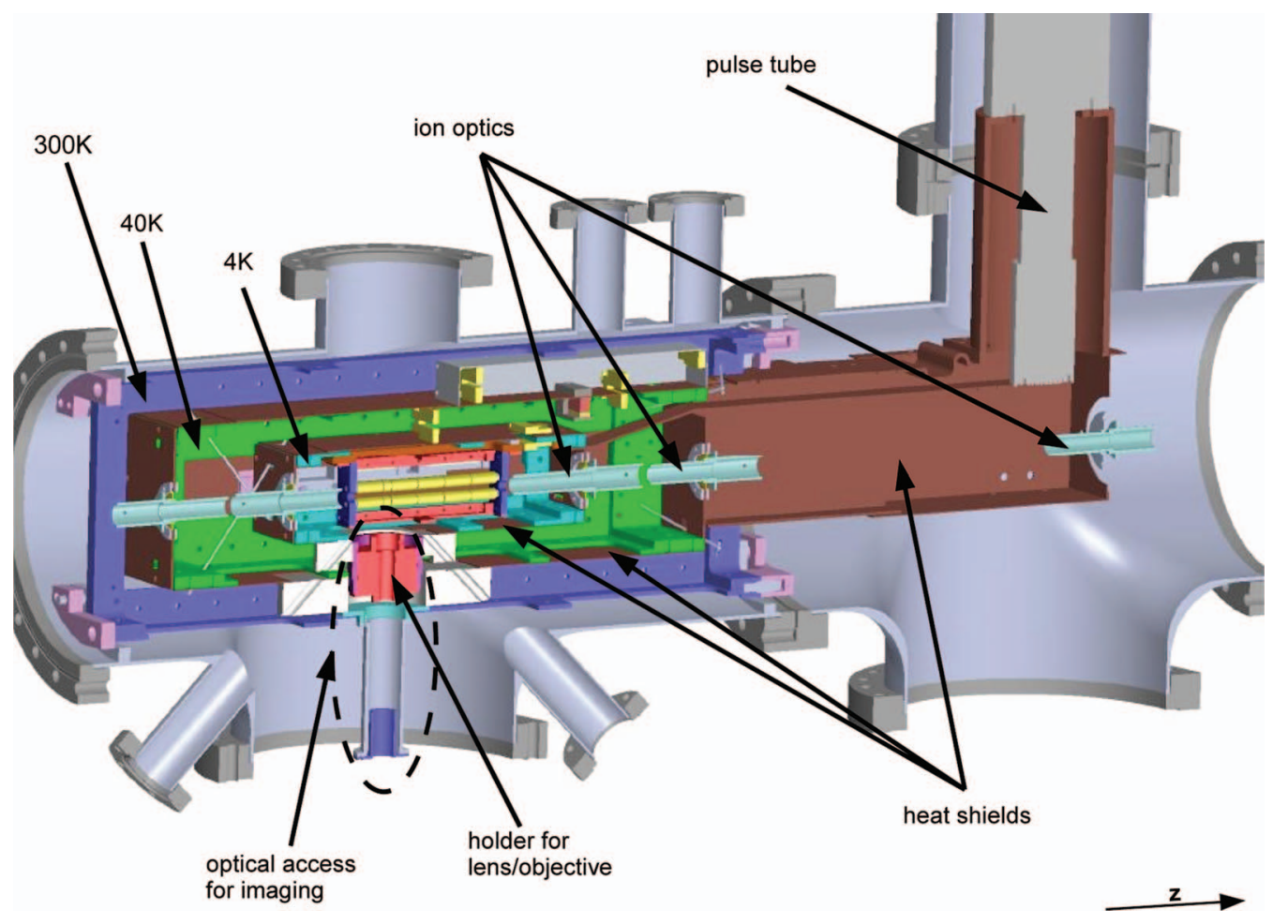

FIG. 1. Vertical cross section along the z-axis of the cryogenic Paul trap CryPTEx.

imaging system with single ion resolution; on-axis access and ion optics for external ion injection into the cryogenic region; provision of a main trap and a pretrap for ion loading; and compatibility with the electrode dimensions and the rf parameters of the proven Aarhus design. ${ }^{18,39,56-60}$ All these specifications have been achieved with the present design.

\section{CRYOGENIC DESIGN AND VACUUM SYSTEM}

\section{A. Cryogenic surroundings}

The first and foremost question to be addressed was the simultaneous need for very good mechanical stability of the electrode arrangement and the cold optical elements as well as the reduction of the thermal load at $4 \mathrm{~K}$ to a level adequate for operation with a pulse tube. As the main construction principle, we have applied a nested spoke-held suspension system which is both compact and stiff. Figure 1 shows a drawing of a section of the setup. The temperature decreases going inwards, starting from the vacuum chamber at room temperature, the $300 \mathrm{~K}$ stage, to the trap at $4 \mathrm{~K}$, with an intermediate stage held at $40 \mathrm{~K}$. These three temperature stages are contained within individual rectangular box-like frames assembled from wire-eroded stainless-steel plates. The steel parts were polished to increase reflectivity and reduce the thermal radiation heat load of these elements. The innermost box (4 K) is suspended from the $40 \mathrm{~K}$ box. This $40 \mathrm{~K}$ box is again suspended from the $300 \mathrm{~K}$ outer frame by means of counteracting spoke assemblies of $10 \mathrm{~cm}$ long, $2 \mathrm{~mm}$ diameter stainlesssteel spokes (see Fig. 2). This minimizes heat conductivity while providing a very high stiffness. There are eight spokes between adjacent temperature stages. Inside of the $4 \mathrm{~K}$ box, an oxygen-free high conductivity (OFHC), high purity (99.99\%) copper plate of $5 \mathrm{~mm}$ thickness supports the different components inside the box, while establishing a very homogeneous temperature distribution.

The $40 \mathrm{~K}$ stage (green frame in Fig. 1) and the $4 \mathrm{~K}$ stage (turquoise frame) are connected to the two temperature stages of a pulse-tube cryocooler with low vibration levels (only 7-9 $\mu \mathrm{m}$ displacement). It is located in a six-way cross chamber attached to the main chamber and it can remove a heat load of up to $40 \mathrm{~W}$ at the $40 \mathrm{~K}$ stage and up to $1 \mathrm{~W}$ at the $4 \mathrm{~K}$ stage. The pulse tube is not directly mounted to the trap vacuum chamber in order to keep the direct lines of sight to the trap center free of obstruction, and to damp vibrations introduced by the cryocooler. Both stages are protected by silverplated OFHC copper shields (shown in brown) with a thickness of $1 \mathrm{~mm}$, which are mounted on the respective stainless steel frames. They provide both a high thermal conductivity at cryogenic temperatures and a high reflectivity for thermal

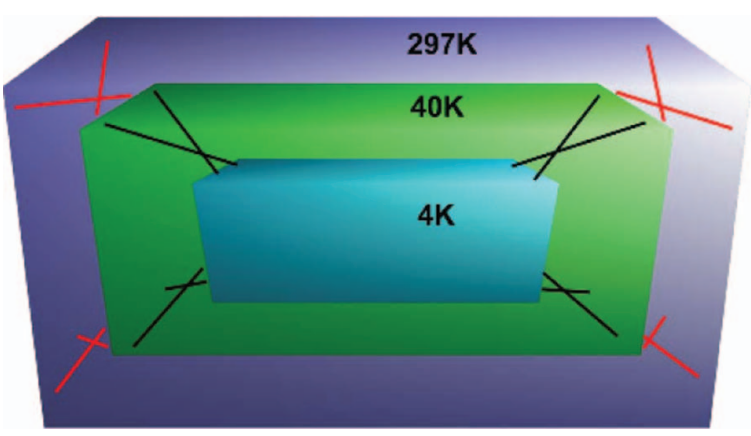

FIG. 2. Schematic drawing of the cryogenic setup consisting of three temperature stages suspended within each other by means of spokes (red and black lines). 
radiation. Each stage is connected to the respective cryocooler stage by silver-plated ultra-high purity OFHC $2 \mathrm{~mm}$ thick sheets. These sheets have corrugations to reduce vibrational coupling between the cryocooler and the $4 \mathrm{~K}$ and $40 \mathrm{~K}$ stages. The whole setup with a total mass of $3 \mathrm{~kg}(4 \mathrm{~K})$ and $6 \mathrm{~kg}$ $(40 \mathrm{~K})$ can be cooled down from room temperature to $4 \mathrm{~K}$ (inner stage) and $35 \mathrm{~K}$ (outer stage) within $15 \mathrm{~h}$. The $300 \mathrm{~K}$ frame (depicted in dark blue) is inserted from one end of the main chamber, plugged into stainless steel holders (shown in pink), and positioned with bolts mounted to both ends of the frame. The whole cryogenic part of the setup can thus be removed as a single unit from the main chamber, a feature particularly convenient for adjusting the different electrodes and optical elements to the required tolerances before insertion into the vacuum chamber. As all the materials used (mainly stainless steel and OFHC copper) have similar thermal expansion coefficients, only minor deformations (well below $100 \mu \mathrm{m}$ ) appear during cool down. A total of four temperature sensors are installed: we use calibrated Cernox-type elements at the $4 \mathrm{~K}$ trap holder, at the $4 \mathrm{~K}$ ground plate, at the coldhead stage connected to it, and one sensor is placed at the $40 \mathrm{~K}$ frame. Their outputs are digitally logged.

\section{B. Optical access}

Optical ports (CF40) located in the horizontal plane of the main vacuum chamber are directed towards the trap center from both sides along the z-axis, under $90^{\circ}, 68^{\circ}$, and $45^{\circ}$ with respect to the trap axis. There are two additional diagonal CF40 ports and one perpendicular CF40 port in the vertical plane through the trap axis. The horizontal CF40 ports for the various laser beams are mounted with $\mathrm{CaF}_{2}$ windows or broadband anti-reflection (AR) coated (range: $260-313 \mathrm{~nm}$ ) fused silica windows. One of the $45^{\circ}$ ports in the horizontal plane is used to introduce a thin optical fiber to the trap center for convenient alignment of the laser beams. This assembly consists of a $2 \mathrm{~cm}$ long fiber with a diameter of $100 \mu \mathrm{m}$ clamped on a $4 \mathrm{~mm}$ diameter stainless steel holder which is mounted to an XYZ manipulator. Adjustments to the position of this fiber are performed with the help of the manipulator in order to bring it to the intended position of the trapped ions at the center of the electrode assembly in the main trap. The imaging system is focused on the tip of this fiber. The fiber tip is employed to align all laser light beams used in the experiment either by monitoring the image or the shadow of the fiber. The fiber is retracted out from between the electrodes to the $40 \mathrm{~K}$ region once this alignment is completed.

A reduction of the black body radiation as well as of residual gas from the room-temperature vacuum entering the $4 \mathrm{~K}$ region is achieved by the use of high aspect ratio apertures between the different temperature regions. In this way, (98 \pm 1$) \%$ of the total solid angle is determined by radiation which is emitted from $4 \mathrm{~K}$ surfaces and only $(2 \pm 1) \%$ of the solid angle consists of BBR generating from room temperature surfaces. Each optical access point in the cryogenic shields is thus designed as a narrow tunnel with a small solid angle for both thermal radiation and ballistic gas particles. These horizontal apertures consist of deep holes in aluminium-milled parts mounted on the thermal shields. The holes have diameters of $5 \mathrm{~mm}$ in the $4 \mathrm{~K}$ shield and $7 \mathrm{~mm}$ in the $40 \mathrm{~K}$ shield. Decreased vacuum conductance leads to a higher efficiency of the differential pumping between the different stages.

Along the central axis there are $100 \mathrm{~mm}$ long stainless steel drift tubes with an inner diameter of $10 \mathrm{~mm}$ mounted on each heat shield. The drift tubes are attached to ring insulators made of sapphire, which are clamped to their respective cryogenic heat shields enabling an efficient cooling of the drift tubes while keeping them electrically isolated at voltages up to $2 \mathrm{kV}$. These electrodes guide and focus ion beams injected in or extracted from the trap.

Furthermore, the sources for atomic and molecular beams needed for the experiments at AU are mounted in two opposite $45^{\circ}$ ports in the horizontal plane. For these two sources, additional elements introduced into the corresponding holes of the aluminum tubes reduce the access diameter down to $1 \mathrm{~mm}$ at the $4 \mathrm{~K}$ shield. This leads to a collimation of the particle beams injected to the trap while preventing the accumulation of deposits of these particles onto the electrodes.

\section{Pumping system}

The vacuum chamber for the trap is connected to a sixfold CF200 cross. The pulse tube cryocooler (SHI, model RP-082B) and the rf-dc coupler circuit with its electrical feedthroughs are attached to this part. Two $300 \mathrm{l} / \mathrm{s}$ turbomolecular pumps with high compression are used, one on each vacuum chamber. The fore-vacuum outlets of these two pumps are connected to a $70 \mathrm{l} / \mathrm{s}$ turbomolecular pump, which is in turn connected to a scroll pump. The compression ratio for hydrogen, a major residual gas component, is effectively multiplied in this way by a factor of more than thousand.

The main chamber can only be baked at a moderate $60^{\circ} \mathrm{C}$ after assembly since many of the built-in components will not tolerate higher temperatures. The large number of surfaces increases the overall pumping resistance which causes the vacuum to remain in the $10^{-8}$ mbar region when the cryocooler is not in operation. However, when the cooling is on, the pressure measured using ionization gauges located at the chamber walls reaches values in the high $10^{-10} \mathrm{mbar}$ range. Within the $40 \mathrm{~K}$ and $4 \mathrm{~K}$ enclosures, cryosorption reduces outgassing and binds residual gas to the cold surfaces efficiently. We have no direct means to measure the pressure in those differentially pumped enclosures. However, extremely suppressed reaction rates and extended ion lifetimes indicate very low residual gas densities in the trap region consistent with the expected cryogenic pumping performance (see Sec. VIII).

A molecular beam of hydrogen is injected into the trap region for the investigation of chemical reactions forming hydride ions. The hydrogen beam originates from a needle valve and is shaped in two small differentially pumped vacuum sections that are separated by a skimmer. Both sections are pumped by $70 \mathrm{l} / \mathrm{s}$ turbomolecular pumps backed by a single scroll pump. A small orifice separates these sections from the main chamber. A similar setup for an atomic beam of magnesium is available. 


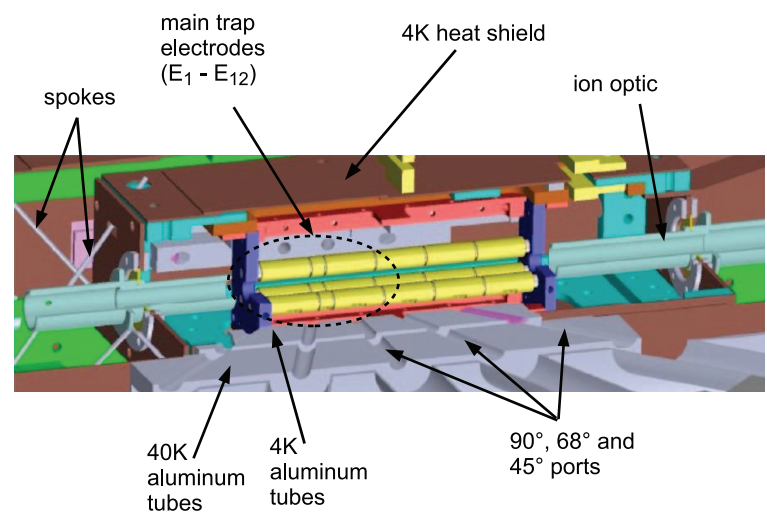

FIG. 3. Cross section through the trap region at $4 \mathrm{~K}$.

\section{TRAP ASSEMBLY}

The trap assembly consists of a total of 24 electrodes. The first 12 electrodes ( $E_{1}$ to $E_{12}$, see Fig. 3) are used to generate the main trapping potential. The other 12 electrodes $\left(E_{13}\right.$ to $\mathrm{E}_{24}$ ) can be used to produce additional trapping potentials in order to extend the trapping region in the axial direction or to create a side trap into which the trapped ions can be shifted and stored. The electrodes all have $20 \mathrm{~mm}$ length (see Fig. 4) except the middle electrodes of the main trap which are shorter with $2 z_{0}=5.4 \mathrm{~mm}$. These dimensions were chosen such that an optimally harmonic potential $\Phi(z)=\eta U_{E C} \frac{z^{2}}{z_{0}^{2}}$ along the trap axis is created, with geometric correction factor $\eta$, potential $U_{E C}$, and axial displacement from the trap center $z$. Numerical simulations (see Fig. 5) have shown that the geometry of the trap, which is based on the dimensions of one of the AU traps, ${ }^{57}$ leads to $\eta=0.259$.

The electrodes are manufactured from $2 r_{e}=8 \mathrm{~mm}$ diameter OFHC copper rods which have been gold plated to enhance the rf conductivity and to reduce patch potentials as well as chemical corrosion. A $10 \mu \mathrm{m}$ thick silver plating between gold and copper surfaces prevents atoms from the $5 \mu \mathrm{m}$ thick gold layer from diffusing into the copper substrate. This plating will guide most of the rf current applied to the electrodes due to the skin effect. The skin depth is about $5 \mu \mathrm{m}$ at a few MHz. The 24 electrodes are hollow. They are mounted on four polished alumina rods with a diameter of $3.8 \mathrm{~mm}$ which are arranged in parallel on a square pattern with their respective axis at a diagonal distance of $15 \mathrm{~mm}$. This leads to a distance of $r_{0}=3.5 \mathrm{~mm}$ between electrode tip and the z-axis of the trap (see Fig. 4). Thin-walled alumina tubes, tightly fitting the rods, are used as spacers between the electrodes. There
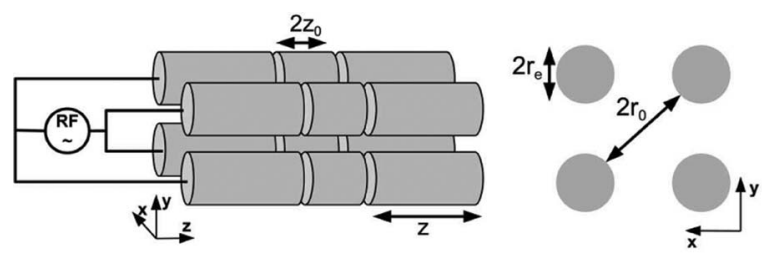

FIG. 4. Drawing of the main trap electrodes (not to scale). A rf voltage of $U=U_{\mathrm{rf}} \cos (2 \pi f t)$ is applied to the electrodes with opposite phase for each electrode set paired diagonally.

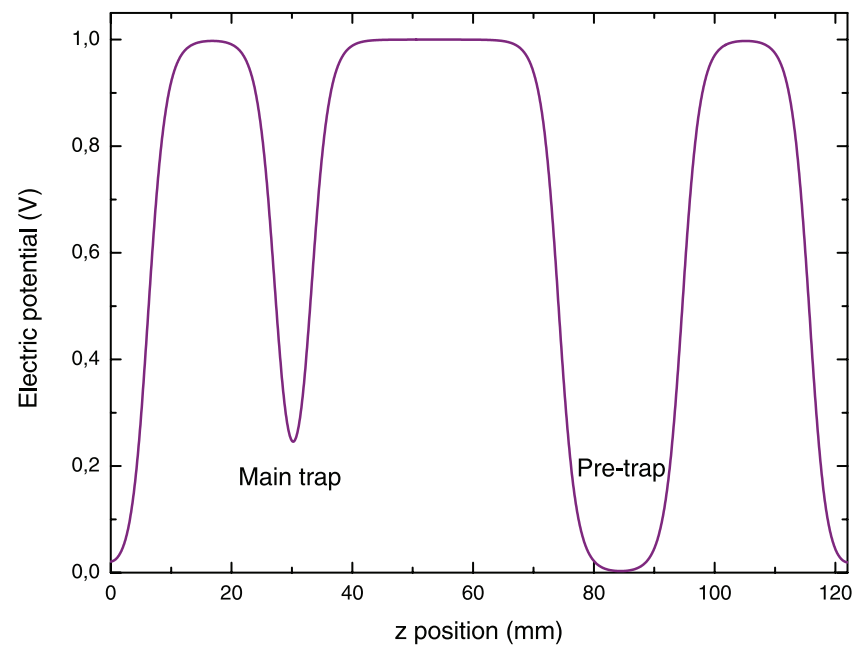

FIG. 5. SIMION (Ref. 61) simulation for the electrical potential along trap axis $(x=y=0)$; the voltage applied between the center and the endcap electrodes is $1 \mathrm{~V}$. The center electrodes of the main trap and the pretrap are grounded.

are only very narrow gaps (500 $\mu \mathrm{m}$ broad, $1 \mathrm{~mm}$ deep) left open between the electrodes on a single rod to avoid charging up of the rods and spacers. The four rods are held by massive copper elements at both ends of the $120 \mathrm{~mm}$ long assembly. These holders have a $7 \mathrm{~mm}$ aperture each on the central axis through which ions can be guided in and out of the trap. The holders are directly mounted on the $4 \mathrm{~K}$ base plate. A stainless steel frame stiffens the assembly.

The electrodes are electrically connected to the dc voltage source and the rf source as well as thermally anchored to the $4 \mathrm{~K}$ base plate by means of $2 \mathrm{~mm}$ diameter ultra-high purity copper wires directly soldered to them. These wires are clamped between two sapphire blocks $(5 \times 8 \times 30 \mathrm{~mm})$ in groups of six wires each. The blocks are pressed firmly onto the $4 \mathrm{~K}$ basis by stainless steel elements (see Fig. 6). The sapphire blocks serve the purpose of electrically isolating the wires and the electrodes from ground, and, at the same time, connecting them thermally to the $4 \mathrm{~K}$ reservoir. This

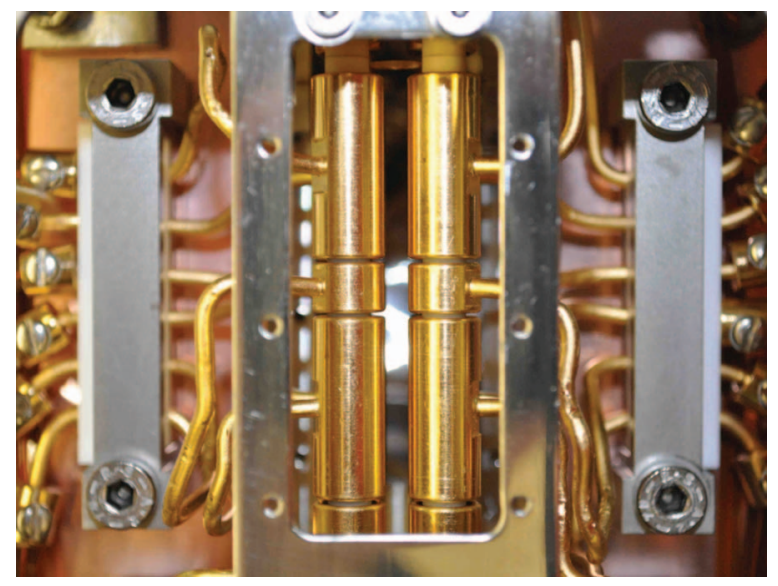

FIG. 6. Photograph of the trapping electrodes before final assembly of CryPTEx. This top view shows the upper six electrodes of the main trap as well as two out of four sapphire clamps for the copper rf feeds. These clamps enable cooling of the electrodes through the rf feeding wires. 
solution allows for the application of large clamping forces to the copper wires in order to optimize thermal contact with the sapphire slabs. The electrodes and the alumina rods holding them are rather free of mechanical stress. Such stress would result in long term failure due to repetitive thermal cycling.

Behind the thermal anchoring clamps the copper wires are connected to two $60 \mathrm{~mm}$ broad ribbon cables, one for each rf phase, which connect them with the rf-dc-coupler. The ribbon cable has a total length of $1.5 \mathrm{~m}$. It is guided through the different temperature stages in a folded pattern with maximized lengths between thermal anchoring points. This minimizes the heat transmission to the $4 \mathrm{~K}$ stage. The ribbon consists of a $50 \mu \mathrm{m}$ thick Kapton layer with 14 copper conductor paths each of $0.4 \mathrm{~mm}$ width and $70 \mu \mathrm{m}$ thickness. Material thickness has been optimized with regard to electrical conductivity, rf heating power dissipation on the cable per unit length, and thermal conductivity. The ends of the ribbon are split in individual leads which are connected to four groups of UHV feedthroughs capable of sustaining operating voltages of $1.5 \mathrm{kV}$.

A voltage of $U= \pm U_{\mathrm{rf}} \cos (2 \pi f t)$, with frequency $f$ $=3.918 \mathrm{MHz}$ and amplitude $U_{\mathrm{rf}} \leq 600 \mathrm{~V}$, is applied to the electrodes. Individual dc voltages of $\pm 100 \mathrm{~V}$ can be superimposed on the rf oscillatory potential on each of the 24 electrodes. These rf and dc voltages are mixed in a coupler circuit (rf-dc-coupler), which is connected to the dc power supplies of the different electrodes. Each channel has its own dc-dc amplifier with a slew rate of $20 \mathrm{~V} / \mu \mathrm{s}$. A frequency generator and a $50 \mathrm{~W}$ rf amplifier feed the coupling circuit through a resonant rising transformer using a ferrite core.

\section{IMAGING SYSTEM}

The system used to image the trapped ions consists of either a Schwarzschild-type reflective microscope objective or an appropriate lens. Different magnifications can be used in this way. The imaging element is mounted directly on the $4 \mathrm{~K}$ assembly inside the vacuum. Aluminium tubes are installed at the different stages to reduce the heat influx into the trap. The real image is projected through an AR-coated fused silica window onto an image intensifier sensitive to UV. The output of this image intensifier is relayed with a photographic objective to a charge-coupled-device (CCD) camera with a size of 1376 pixel $\times 1040$ pixel and with a pixel size of $6.45 \mu \mathrm{m} \times 6.45 \mu \mathrm{m}$. The imaging setup uses the bottom CF200 port in the main vacuum chamber. The image intensifier and CCD camera are mounted underneath this port by means of a manipulator and a bellows that suppresses stray light. For maximum magnification $(\times 15)$ and resolution a reflective objective is used with $23 \mathrm{~mm}$ working distance and $13 \mathrm{~mm}$ effective focal length. The positions of individual ions can easily be resolved (see Fig. 15). The imaging system employing this reflective objective has the additional advantage of being fully achromatic. Alternatively, when a wider field of view is desired, a 1/2 in. diameter convex lens with a focal length of $f=40 \mathrm{~mm}$ is used. A $6 \mathrm{~mm}$ diameter aperture is placed in front of the lens to minimize spherical aberrations. Crystals containing many $10^{4}$ ions can be imaged comfortably although there is no fundamental limitation preventing the trapping and imaging of crystals in CryPTEx that contain several orders of magnitude more ions. Both lens and mirror objective can be mounted on a movable holder placed directly on the $4 \mathrm{~K}$ frame. With the lens, a magnification of about $\times 9$ is achieved. The magnification can be determined by imaging the $100 \mu \mathrm{m}$ diameter quartz fiber. The image intensifier allows for gating times as short as $100 \mathrm{~ns}$ at repetition rates of $10 \mathrm{kHz}$.

\section{INITIAL PERFORMANCE TESTS AS A MASS FILTER: MAPPING OF THE STABILITY REGION TRANSMISSION RESONANCES}

The apparatus is intended for being loaded with HCIs produced by external sources such as an electron beam ion trap (EBIT). For this reason it has been equipped with drift tubes along the z-axis. A small ancillary ion source was set up in order to be able to benchmark the trap performance and the injection performance without the requirement of a dedicated laser system. The transmission of the ions produced there through the trapping region was investigated as a function of the different rf and dc parameters.

The ion beam exits the source with a kinetic energy between $40 \mathrm{eV}$ and $100 \mathrm{eV}$ and is guided to the Paul trap. The vacuum chamber of the Paul trap is directly attached to the source along the common central axis. Ion transmission is monitored by a microchannel plate (MCP) detector on the other end of the Paul trap chamber. The MCP delivers single pulses for each ion hitting it. This enables a counter to register the ion flux.

LabVIEW control software was used to explore the stability region of the mass filter. Measurements of the ion transmission showed distinctive features which were dependent on the $a / q$ reduced parameters, and hence on the ratio of the dc and rf voltages applied to the electrodes on the four rods following the usual definitions (cf. Ref. 3):

$$
a=\frac{4 e U_{\mathrm{dc}}}{m r_{0}^{2}(2 \pi f)^{2}}, \quad q=\frac{2 e U_{\mathrm{rf}}}{m r_{0}^{2}(2 \pi f)^{2}},
$$

where $U_{\mathrm{dc}}$ is the potential used in the usual manner of a quadrupole mass analyzer. The different parameters were scanned repetitively, and two-dimensional $(a, q)$ maps were recorded. Figure 7 shows the results obtained with $\mathrm{He}^{+}$ions. The transmission was determined for $\mathrm{He}^{+}$ions at a kinetic energy of $47 \mathrm{eV}$ and $100 \mathrm{eV}$. As the ion source only reaches a pressure of some $10^{-8}$ mbar there are additional features in the data arising from singly charged ions of its typical residual gas components of the ion source (within the gray shaded area: $\mathrm{N}^{+}, \mathrm{N}_{2}^{+}, \mathrm{O}^{+}$, and $\mathrm{O}_{2}^{+}$). Transmission peaks in structures strongly resemble those seen in the classical Paul trap (for more information see Ref. 2). No attempt at absolute calibration of the transmission efficiency was made. The goal of these measurements was to obtain a qualitative understanding of the mass filter properties of the linear Paul trap. In fact, the observed features correspond to stable trajectories with discrete number of oscillations within the trap rods longitudinal extension. These stable trajectories imply a periodically refocussing effect of the initially divergent paths of ions entering the trap region onto the exit aperture (see Fig. 8). This 

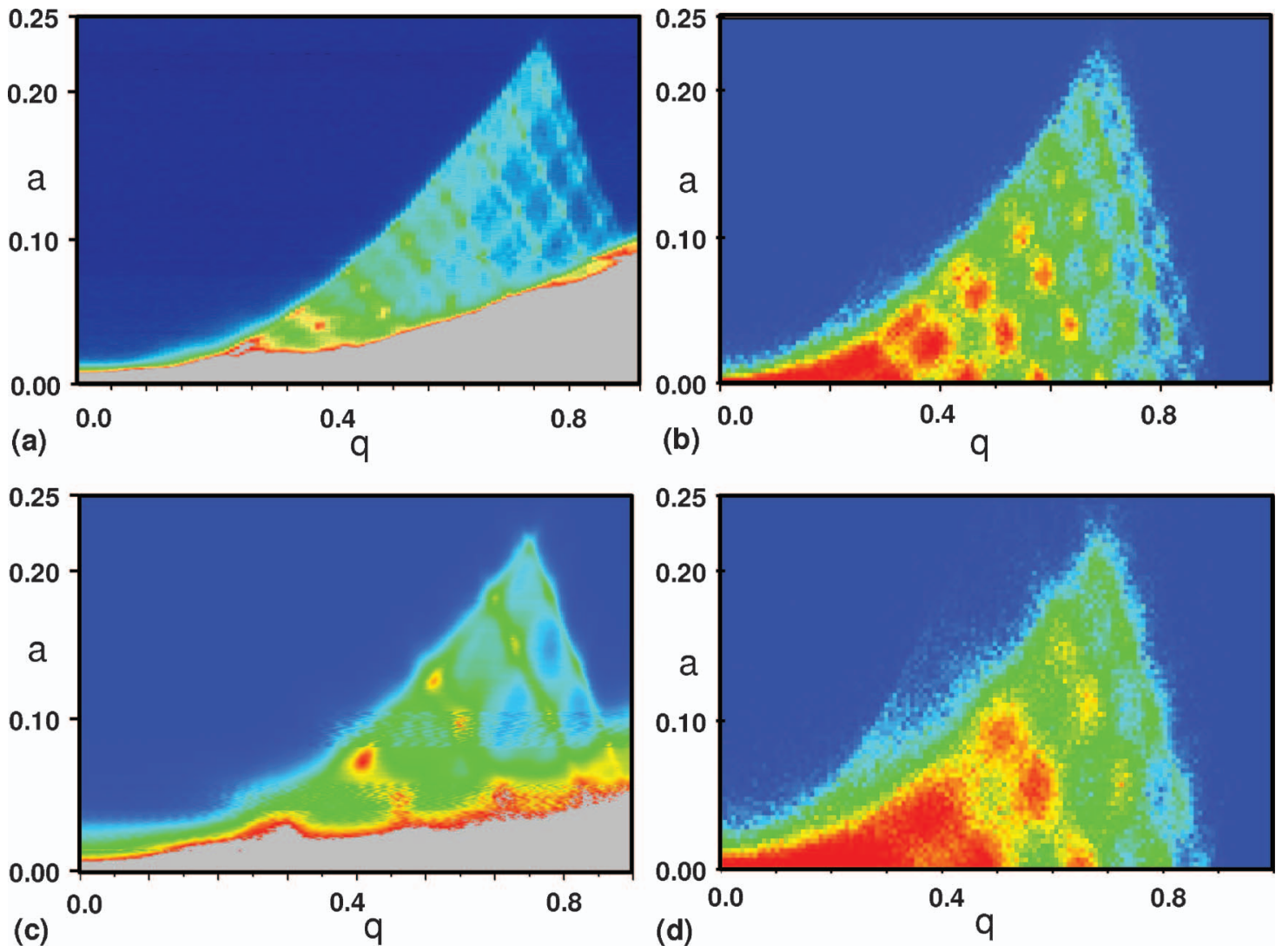

FIG. 7. Transmission efficiency of the mass filter. ${ }^{61,62}$ (a) Experiment: integrated signal on the MCP of transmitted $\mathrm{He}^{+}$ions at $47 \mathrm{eV}$ (in arbitrary units: MCP background 450 (blue) to 45000 (red)); (b) simulation: $\mathrm{He}^{+}$ions at $47 \mathrm{eV}$ (in arbitrary units from 0 (blue) to 100 (red)); (c) experiment: integrated signal on the $\mathrm{MCP}$ of $\mathrm{He}^{+}$ions at $100 \mathrm{eV}$ (in arbitrary units: MCP background 650 (blue) to 5500 (red)); (d) simulation: $\mathrm{He}^{+}$ions at $100 \mathrm{eV}$ (arbitrary units: 0 (blue) to 100 (red)). Ions from residual gas components of the ion source such as $\mathrm{N}^{+}, \mathrm{N}_{2}^{+}, \mathrm{O}^{+}$, and $\mathrm{O}_{2}^{+}$appear in gray shaded areas in the experimental data.

effect could be further studied by means of ion optics tracking simulations carried out with the ion optics modeling program SIMION (Refs. 61 and 62) (see Fig. 7). A model of the trap assembly was generated by exporting the dimensions directly from the SolidWorks program to the SIMION program. The transmission of singly charged $\mathrm{He}^{+}$ions was then simulated for the same kinetic energies, $47 \mathrm{eV}$ and $100 \mathrm{eV}$, that were used to obtain the experimental data. The mass resolution $m / \Delta m$ achieved near the tip of the stability diagram was well above 100 . Given the limited length of the trap, this result approaches the expected performance and indicates that mechanical and electrical specifications have been reached.

These initial tests helped us to debug the apparatus and to calibrate the operating parameters. We demonstrated the proper functioning of the system by displaying resonant fea-

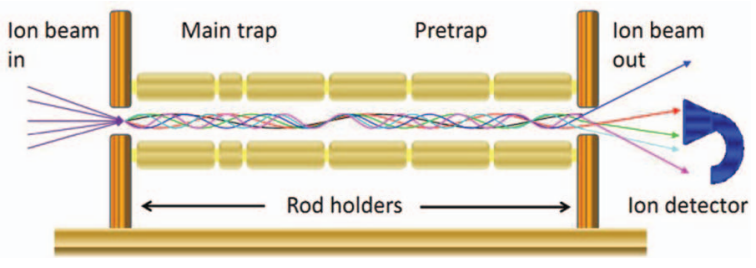

FIG. 8. Effect of the pseudo-potential on the ion beam trajectories. ${ }^{62}$ Depending on the number of nodes different exit beam divergences are obtained. Detection efficiency is affected by this divergence (see Fig. 7) and shows therefore periodic structures parametrized by the trap fields. tures linked to the pseudo-potential of the trap. The ion injection system crucial to future HCI work was also implemented. The trap setup was upgraded with four optical breadboards after the initial commissioning experiments, and the apparatus was moved to Aarhus University for performance tests with Doppler laser cooled trapped ions.

\section{PHOTOIONIZATION AND DOPPLER LASER COOLING}

The trap was commissioned with ${ }^{24} \mathrm{Mg}^{+}$ions. Ions are loaded by laser photoionization ${ }^{63}$ at the trap center from a thermal atomic beam generated by means of an evaporative source. The translational degrees of freedom of the trapped $\mathrm{Mg}^{+}$ions are then laser cooled through the fast cycling optical transitions in $\mathrm{Mg}^{+}$. The $3 s^{2} S_{1 / 2} \leftrightarrow 3 p^{2} P_{3 / 2}$ line in $\mathrm{Mg}^{+}$at $279.6 \mathrm{~nm}$ (see Fig. 9) is particularly suited for laser cooling. With the right cooling conditions, the ions cool down to a few tens of $\mathrm{mK}$ and arrange themselves in crystalline structures known as Coulomb crystals. The imaging system detects the spontaneous emission of the Doppler-cooled $\mathrm{Mg}^{+}$ions. Their spatial distribution can be monitored in real time as the cooling laser light is applied during the complete experimental cycle.

Laser systems suitable for both cooling and photoionization are available at Aarhus University. Brief descriptions of these laser setups are given below (also see Figs. 10 and 11). 

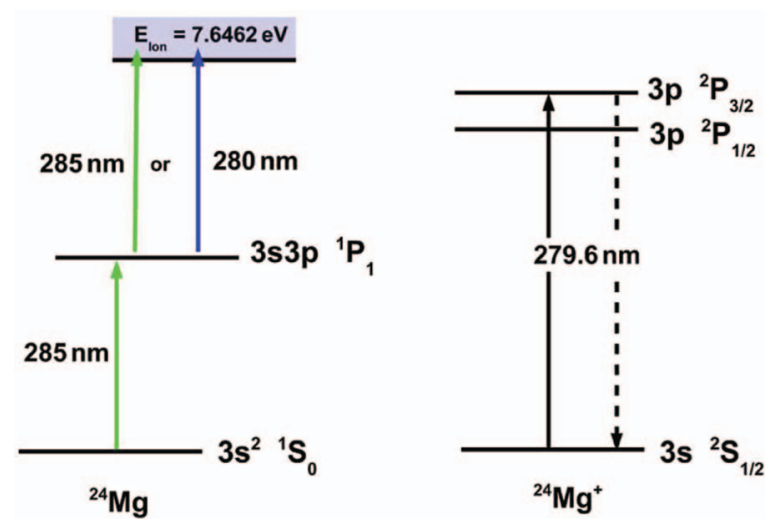

FIG. 9. Energy level schemes of ${ }^{24} \mathrm{Mg}$ atoms, relevant for photoionization, and ${ }^{24} \mathrm{Mg}^{+}$ions for Doppler laser cooling.

\section{A. Photoionization laser}

The ionization energy of $\mathrm{Mg}$ is $7.65 \mathrm{eV}$. In a two-step process, absorption of a $285 \mathrm{~nm}$ photon first excites the atoms from the $3 s^{2}{ }^{1} S_{0}$ state to the $3 s 3 p^{1} P_{1}$ state from where it can be ionized in a second step by absorbing either another $285 \mathrm{~nm}$ photon or a $280 \mathrm{~nm}$ photon from the cooling laser beam (see Fig. 9). The first step is isotope selective, suppressing the production of ${ }^{25} \mathrm{Mg}^{+}$and ${ }^{26} \mathrm{Mg}^{+}$. A continuous wave (cw) ring dye laser (Coherent 699) pumped by an argon ion laser (Spectra physics) generates narrow bandwidth $570 \mathrm{~nm}$ radiation. This light is frequency doubled in an external bow-tie cavity incorporating an BBO crystal and locked using a HänschCouillaud scheme. ${ }^{64}$ Laser powers of typically $10-30 \mathrm{~mW}$ at $285 \mathrm{~nm}$ are achieved at sufficiently narrow line width. The laser beam is focused at the trap location (see Fig. 10). A Mg atomic source (Alva source type AS-3-Mg-50V from alvatec) is heated to a few hundred degrees centigrade by a current of about $6 \mathrm{~A}$ in order to evaporate $\mathrm{Mg}$ atoms. An atomic beam is produced by a $1 \mathrm{~mm}$ diameter aperture between source and main chamber and by the additional aperture in the $4 \mathrm{~K}$ shield. This beam is aligned toward the trap center where the atoms

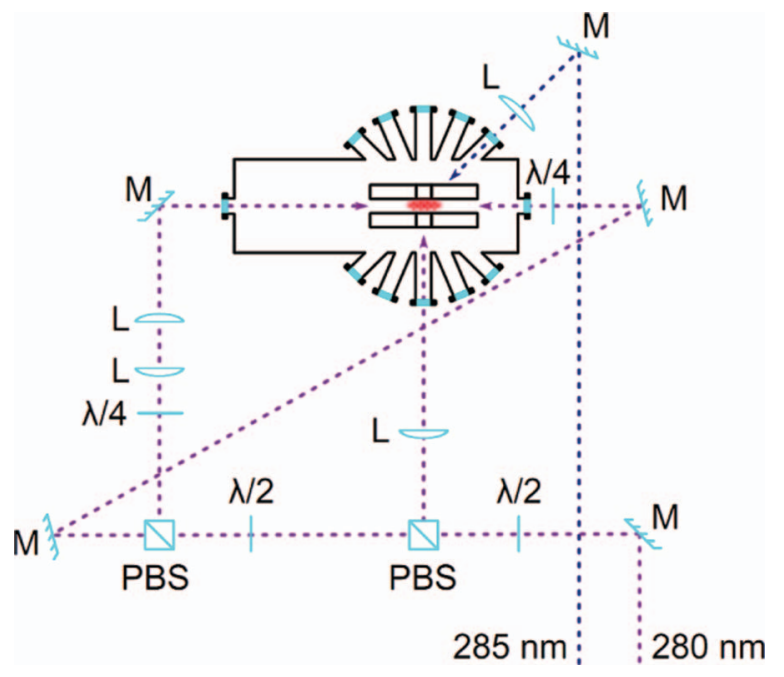

FIG. 10. Laser setup for photoionization and Doppler cooling of $\mathrm{Mg}^{+} .(\lambda / 2)$ and $(\lambda / 4)$, retardation wave-plates; mirror $(\mathrm{M})$; lenses $(\mathrm{L})$; and polarizing beam splitter (PBS).

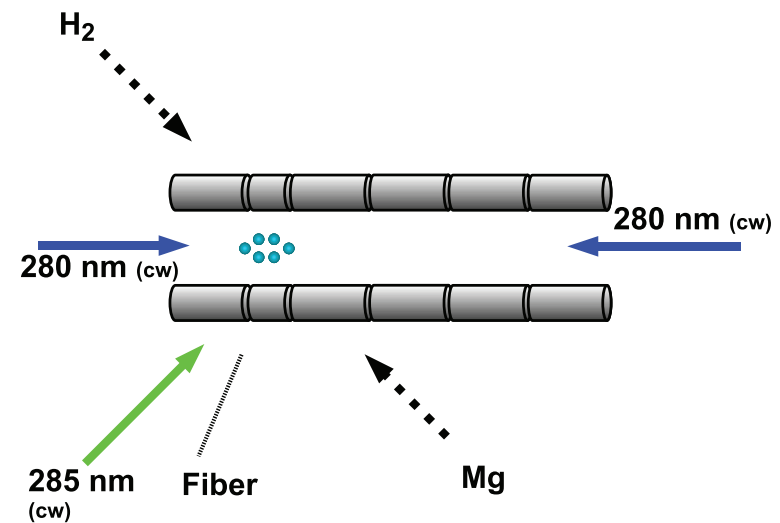

FIG. 11. Schematic drawing of the laser setup used to test the trap performance showing the cooling laser and the photoionization laser beams as well as the atomic and molecular beams.

are photoionized. With kinetic energies well below $1 \mathrm{eV}$, the $\mathrm{Mg}^{+}$ions are instantly trapped in the effective potential of the Paul trap.

\section{B. Cooling laser}

The Doppler cooling laser beam at $280 \mathrm{~nm}$ is generated via frequency quadrupling of the output of a fiber laser (NKT Photonics/Koheras), which generates up to $2.4 \mathrm{~W}$ of light at $1118 \mathrm{~nm}$ wavelength. Laser radiation at $559 \mathrm{~nm}$ is obtained via resonant frequency doubling in an external bow-tie cavity. It incorporates an LBO crystal and is locked using the PoundDrever-Hall technique. ${ }^{65}$ Further doubling to $280 \mathrm{~nm}$ is performed in a second bow-tie cavity using a BBO crystal. This cavity is locked using a Hänsch-Couillaud scheme. ${ }^{64}$ Laser powers up to $100 \mathrm{~mW}$ at $280 \mathrm{~nm}$ could be achieved at line widths well below $1 \mathrm{MHz}$. This UV beam is branched into two beams, which are guided into the trap region to counterpropagate, linearly polarized, along the trap axis. The intensity balance is controlled using a polarizing beam splitter and a half-waveplate (see Fig. 10). The laser power at the trap location was varied between $1-10 \mathrm{~mW}$ per arm. This beam is focused down to a diameter of roughly half a millimeter at the trap center.

\section{A compact laser system for sympathetic cooling of $\mathrm{HCls}$ with large $\mathrm{Be}^{+}$crystals}

CryPTEx provides an excellent environment for trapping and investigating HCIs. Since HCIs have no suitable laser cooling transitions they have to be sympathetically cooled by an ion species with a similar charge-to-mass ratio. ${ }^{67}$ For that purpose, ${ }^{9} \mathrm{Be}^{+}$is chosen. Here, the $2 s^{2} S_{1 / 2} \leftrightarrow 2 p^{2} P_{3 / 2}$ transition at $313 \mathrm{~nm}$ wavelength can be driven as closed-cycle cooling transition. Large ${ }^{9} \mathrm{Be}^{+}$crystals are required for high sympathetic cooling efficiencies. In order to achieve the necessary laser intensity over a large ion crystal volume, a high optical power is required. We have built a solid state laser source (see Fig. 12) that delivers up to $730 \mathrm{~mW}$ at the required wavelength. The setup of the laser system is based on Ref. 68. The obtainable power enables large beam diameters and hence can sustain large ${ }^{9} \mathrm{Be}^{+}$crystals. The fundamental wavelengths of $1051 \mathrm{~nm}$ and $1550 \mathrm{~nm}$ are each 


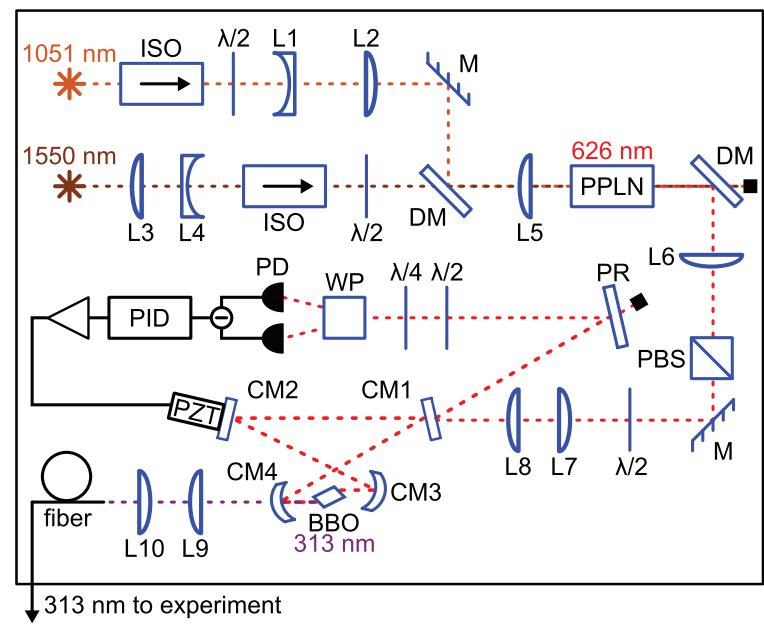

FIG. 12. Schematic diagram of $313 \mathrm{~nm}$ laser setup. Faraday isolator (ISO), $(\lambda / 2)$ and $(\lambda / 4)$ retardation wave-plates, mirror (M), dichroic mirror (DM), lenses (L1-L9), Wollaston prism (WP), photodiode (PD), piezoelectric transducer (PZT), curved mirrors (CM), partial reflector (PR), polarizing beam splitter (PBS), periodically-poled lithium niobate (PPLN), and beta-barium borate (BBO) crystals.

created by a $5 \mathrm{~W}$ fiber laser and amplifier system (NKT Photonics/Koheras). The sum frequency of these two wavelengths is generated inside a $40 \mathrm{~mm}$ long periodically poled lithium niobate (PPLN) crystal (Covesion) for quasi phasematching with a grating period of $10.95 \mu \mathrm{m}$ at a temperature of $193.6 \pm 0.01{ }^{\circ} \mathrm{C}$. With this method, a laser power of $1.8 \mathrm{~W}$ at $626 \mathrm{~nm}$ wavelength is generated. Subsequently, the final wavelength of $313 \mathrm{~nm}$ is obtained by cavity-enhanced frequency doubling inside a BBO crystal (Döhrer). The cavity is of bow-tie type and uses a Hänsch-Couillaud ${ }^{64}$ lock. In order to make the setup easily transportable, the laser system is mounted on an actively isolated breadboard support (Thorlabs) that measures $75 \times 90 \mathrm{~cm}^{2}$. The fiber laser systems are integrated in the support. A photonic crystal fiber for $313 \mathrm{~nm}$ provides a flexible link to CryPTEx.

\section{LIFETIME OF TRAPPED IONS}

The ion loss rate is an important characterization of the quality of the trapping fields, as well as a tool to determine the vacuum conditions. Ion loss from the trap can occur through ion-ion or ion-neutral (background) collisions that transfer the participant ion(s) into unstable orbits. Careful adjustment of the rf and dc trapping fields can increase the lifetime of the crystal to many hours, or even days, if UHV conditions are met. We measure the lifetime of the fluorescent ions in the trap after optimizing the trapping potentials. Our imaging system and data acquisition enable a careful tracing of the exact number of fluorescing ions in the trap. The calibrated imaging system yields a high-resolution image of the spheroidal shape from which the crystal volume can be obtained (see upper image in Fig. 13). The number of trapped ions is then derived from the crystal volume using the calculated ion density for given trap potentials. The upper trace in Fig. 14 depicts the number of fluorescent ions in the cloud as a function of time. A fit to the data yields the lifetime of the trapped ions; it is of the order of $28 \mathrm{~h}$. The main constituent of the background gas

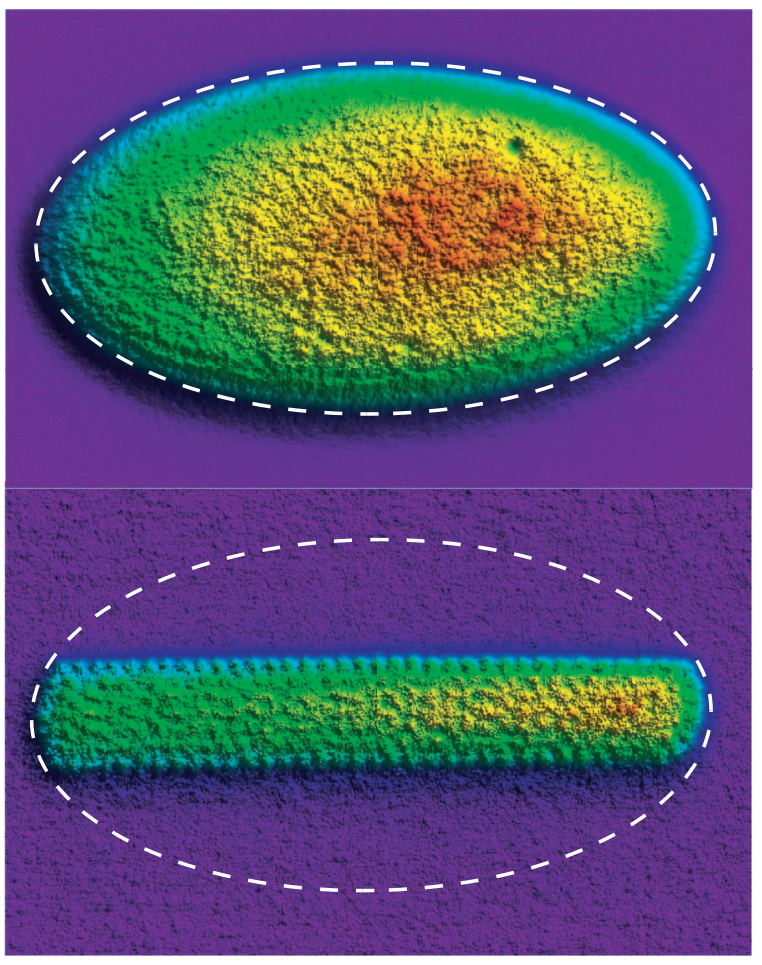

FIG. 13. A larger field of view is obtained with a $40 \mathrm{~mm}$ UV lens (uncoated). The upper CCD fluorescence image depicts a pure $\mathrm{Mg}^{+}$crystal with $\sim 10^{4}$ ions. In both images, the fluorescence intensity is shown in arbitrary units from 50(blue) to 1000 (red). The length of this crystal is $1 \mathrm{~mm}$ with aspect ratio 0.5 . The deviation from the standard spheroidal shape visible in the lower CCD fluorescence image is caused by the (intentional) presence of $8 \times$ $10^{3} \mathrm{MgH}^{+}$dark ions. The bi-crystal is still completely spheroidal.

in the cryogenic environment of the trap is molecular hydrogen. A sensitive tool for the determination of the vacuum conditions is the determination of the rate at which $\mathrm{MgH}^{+}$ions are formed. This process takes place through a photochemical reaction

$$
\mathrm{Mg}^{+}\left(3 p^{2} P_{3 / 2}\right)+\mathrm{H}_{2} \rightarrow \mathrm{MgH}^{+}+\mathrm{H},
$$

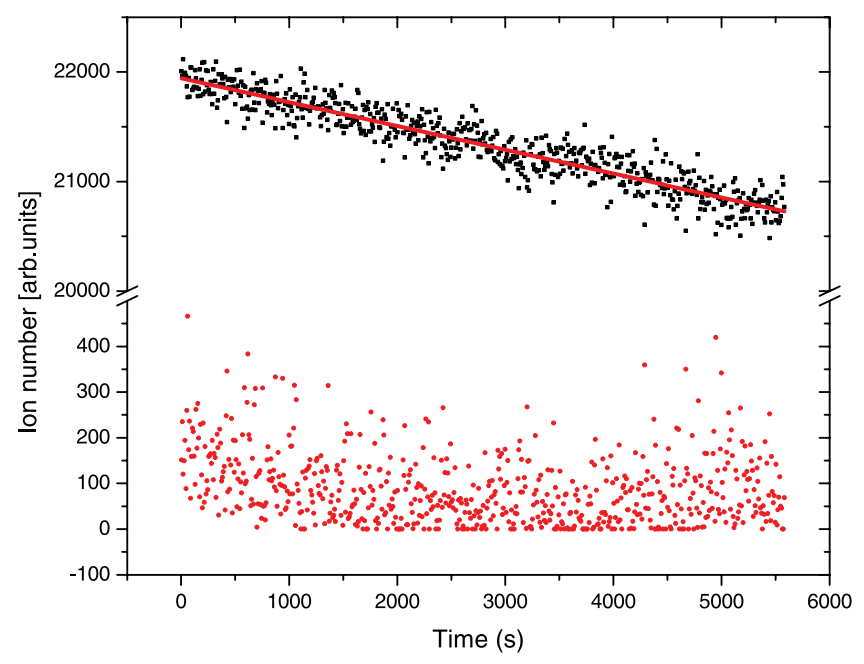

FIG. 14. Graph depicting the number of fluorescing $\mathrm{Mg}^{+}$ions (upper trace) and the number of dark ions (lower trace) versus time. The red line represents a linear fit to the data from which the decay time constant is obtained. 
whereby it should be noted that the reaction rate is proportional to the population of the excited $3 p^{2} P_{3 / 2}$ state. ${ }^{66}$ Keeping the degree of ion excitation constant, the reaction rate is directly proportional to the number density of hydrogen at the position of the $\mathrm{Mg}^{+}$ions. The $\mathrm{MgH}^{+}$ions do not scatter light from the cooling laser light. These ions form an outer dark shell around a fluorescing cigar-shaped $\mathrm{Mg}^{+}$ion center (see Fig. 13) due to their smaller charge-to-mass ratio. ${ }^{66}$ The number of dark ions can be accurately determined by subtracting the observed number of fluorescing ions from the total ion count (see, e.g., Ref. 46). This total ion count is in turn obtained from the length of the crystal given by the fluorescing ions and the aspect ratio that was calibrated on a pure $\mathrm{Mg}^{+}$ crystal.

The aspect ratio is essentially unaffected by the presence of $\mathrm{MgH}^{+}$, as is illustrated in Fig. 13, where length and aspect ratio are unchanged even after more than two-thirds of the light ions have reacted with hydrogen (also see Ref. 46) to form $\mathrm{MgH}^{+}$. The aspect ratio is also independent of crystal size. The lower trace in Fig. 14 depicts the number of nonfluorescent (dark) ions in the cloud as a function of time. No significant change in dark ion number is observed over $2 \mathrm{~h}$ of storage. The statistical accuracy of the dark ion count, and the changes therein, are limited. Instead, we use the decay time constant of the fluorescent ions obtained above (upper trace in Fig. 14) to obtain a conservative upper bound of the reaction rate. Other, unknown, mechanisms of ion loss may well be present but do not affect the determination of an upper limit in the reaction rate. By comparing this reaction rate (i.e., at $1 / 28 \mathrm{~h}^{-1}$ ) in the present trap with that in another ion trap ${ }^{46}$ in a room temperature chamber that is otherwise exactly identical to ours (aside from the $4 \mathrm{~K}$ trapping environment) with known density of hydrogen, we determined a conservative upper bound on the hydrogen pressure of few $10^{-13} \mathrm{mbar}$ (if dominated by $300 \mathrm{~K}$ particles) or $10^{-15}$ mbar (if dominated by $4 \mathrm{~K}$ particles). There are indications that this pressure is mainly the result of ballistic gas influx through the laser apertures. In the future, AR-coated windows will be installed on the heat shields to block this undesired gas input and further reduce the residual gas density.

The images depicted in Figs. 13 and 15 were taken from crystals residing in the main trap. By the application of suitable voltages the crystal can be smoothly transported from the

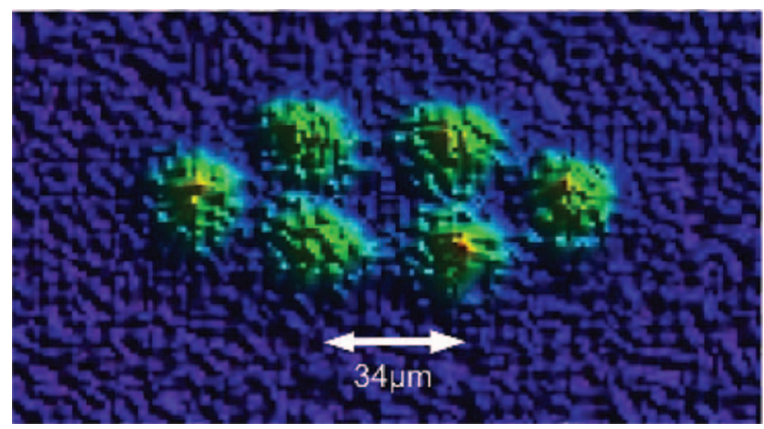

FIG. 15. CCD fluorescence image taken from a crystal consisting of six $\mathrm{Mg}^{+}$ ions. The intensity is shown in arbitrary units from 50(blue) to 1000 (red). The typical distance between the trapped ions is about $34 \mu \mathrm{m}$. Single ion resolution is achieved with the Schwarzschild mirror objective. main trap region towards the pretrap, where they can be stably stored and laser-cooled. By this means, the crystal is moved from the position where it is exposed to atomic beams or BBR fields. Such transport has been experimentally demonstrated in CryPTEx. The smoothness of the transfer was optimized both by avoiding melting of the crystal during the process as well as by eliminating ion losses. The installation of a lowpass filter for the dc trap potential proved to be indispensable. With these modifications, no ion loss was discernible after 70 shuttling attempts. Also, no ion loss was present with 15 min of storage time in the secondary trapping position.

\section{CONCLUSION}

A cryogenic Paul trap operating at ultra-high vacuum conditions suitable for the trapping of HCIs has been constructed. All specifications and requirements were met with the MPIK design presented in this paper. The trap CryPTEx is stably operated at $4 \mathrm{~K}$ at low thermal loads enabling the $40 \mathrm{~K}$ shield to remain at $35 \mathrm{~K}$. Ultra-high vacuum at an $\mathrm{H}_{2}$ partial pressure of a few $10^{-15}$ mbar (at $4 \mathrm{~K}$ ) has been achieved in the cryogenic trap. Radio frequency voltages up to $1000 \mathrm{~V}$ can easily be sustained. Vacuum ports, 15 of them in total, provide ample optical access to the trap center needed for quantum manipulation of the trapped ions. The low solid angle optical ports provide this access without compromising on the exposure to BBR fields. Black body radiation exposure at $4 \mathrm{~K}$ and $300 \mathrm{~K}$ is $98 \pm 1 \%$ and $2 \pm 1 \%$, respectively. Differential pumping of these access ports is maximized by the design employing low conductance tubes in $4 \mathrm{~K}$ and $40 \mathrm{~K}$ stages. Even lower exposure to BBR fields is made possible in the pretrap.

Ions have been shown to be able to travel without any detectable loss between the main trap and this pretrap. Excellent trap performance has been demonstrated in experiments employing the setup as a mass filter as well as in experiments using laser-cooled $\mathrm{Mg}^{+}$ions forming Coulomb crystals. Coulomb crystals consisting of one component (in our case $\mathrm{Mg}^{+}$ions) as well as two component crystals (consisting of $\mathrm{Mg}^{+}$and molecular $\mathrm{MgH}^{+}$ions) can be generated, trapped, and stored for periods of time of tens of hours.

The design of CryPTEx is not only suitable for high precision spectroscopy on HCIs but is also of particular interest for the preparation of polar molecular ions in defined, or even single, quantum states utilizing the low exposure to BBR in the cryotrap. This low exposure enables measurements with very long coherence times. Experiments towards this end are currently being performed at Aarhus University using CryPTEx. Preliminary results indeed indicate a low exposure to BBR fields.

In the near future, the trap will be coupled to a new EBIT at MPIK, where it will be loaded with HCIs that will be sympathetically cooled by $\mathrm{Be}^{+}$ions. An all-solid state laser system for Doppler laser cooling at $313 \mathrm{~nm}$ wavelength was constructed for this purpose at PTB. The flexible and easily transportable design of CryPTEx enables future experiments on laser and $\mathrm{x}$-ray spectroscopy and manipulation of trapped ions in well-defined quantum states at cryogenic temperatures. 


\section{ACKNOWLEDGMENTS}

M.S. and A.W. gratefully acknowledge funding from the STSM grant of the COST Action IOTA. We thank the MPIK mechanical workshops, in particular the apprentice workshop under direction of S. Flicker, for the competent construction of crucial elements of CryPTEx. The Aarhus University team furthermore appreciate significant support through the Danish National Research Foundation Center for Quantum Optics (QUANTOP), the Danish Agency for Science, Technology and Innovation, the Carlsberg Foundation as well as the Lundbeck Foundation. P.O.S. is supported by DFG through QUEST.

${ }^{1}$ W. Paul and M. Raether, Z. Phys. 140, 262 (1955).

${ }^{2}$ W. Paul, H. P. Reinhard, and U. von Zahn, Z. Phys. 152, 143 (1958).

${ }^{3}$ W. Paul, Rev. Mod. Phys. 62, 531 (1990).

${ }^{4}$ P. Ghosh, "Paul Trap confinement," in Ion Traps (Oxford University Press, 1995), Chap. 2.

${ }^{5}$ G. Werth, Lect. Notes Phys. 749, 31 (2008).

${ }^{6} \mathrm{~S}$. Willitsch, M. T. Bell, A. D. Gingell, and T. P. Softley, Phys. Chem. Chem. Phys. 10, 7200 (2008).

${ }^{7}$ G. Werth, V. N. Gheorghe, and F. G. Major, Charged Particle Traps II (Springer, Berlin/ Heidelberg, 2009).

${ }^{8}$ D. Leibfried, R. Blatt, C. Monroe, and D. Wineland, Rev. Mod. Phys. 75, 281 (2003).

${ }^{9}$ H. Häffner, C. F. Roos, and R. Blatt, Phys. Rep. 469, 155 (2008).

${ }^{10}$ R. Blatt and D. Wineland, Nature (London) 453, 1008 (2008).

${ }^{11}$ P. Gill, Metrologia 42, S125 (2005).

${ }^{12}$ T. Rosenband, D. B. Hume, P. O. Schmidt, C. W. Chou, A. Brusch, L. Lorini, W. H. Oskay, R. E. Drullinger, T. M. Fortier, J. E. Stalnaker, S. A. Diddams, W. C. Swann, N. R. Newbury, W. M. Itano, D. J. Wineland, and J. C. Bergquist, Science 319, 1808 (2008).

${ }^{13}$ D. J. Wineland, R. E. Drullinger, and F. L. Walls, Phys. Rev. Lett. 40, 1639 (1978).

${ }^{14}$ W. M. Itano and D. J. Wineland, Phys. Rev. A 25, 35 (1982).

${ }^{15}$ F. Diedrich, J. C. Bergquist, W. M. Itano, and D. J. Wineland, Phys. Rev. Lett. 62, 403 (1989).

${ }^{16}$ J. D. Prestage, A. Williams, L. Maleki, M. J. Djomehri, and E. Harabetian, Phys. Rev. Lett. 66, 2964 (1991).

${ }^{17}$ M. G. Raizen, J. M. Gilligan, J. C. Bergquist, W. M. Itano, and D. J. Wineland, Phys. Rev. A 45, 6493 (1992).

${ }^{18}$ M. Drewsen, A. Mortensen, R. Martinussen, P. Staanum, and J. L. Sørensen, Phys. Rev. Lett. 93, 243201 (2004).

${ }^{19}$ P. F. Staanum, K. Højbjerre, R. Wester, and M. Drewsen, Phys. Rev. Lett. 100, 243003 (2008).

${ }^{20}$ K. Højbjerre, D. Offenberg, C. Z. Bisgaard, H. Stapelfeldt, P. F. Staanum, A. Mortensen, and M. Drewsen, Phys. Rev. A 77, 030702 (2008).

${ }^{21}$ C. W. Chou, D. B. Hume, J. C. J. Koelemeij, D. J. Wineland, and T. Rosenband, Phys. Rev. Lett. 104, 070802 (2010).

${ }^{22}$ D. J. Larson, J. C. Bergquist, J. J. Bollinger, W. M. Itano, and D. J. Wineland, Phys. Rev. Lett. 57, 70 (1986).

${ }^{23}$ P. Bowe, L. Hornekær, C. Brodersen, M. Drewsen, J. S. Hangst, and J. P. Schiffer, Phys. Rev. Lett. 82, 2071 (1999).

${ }^{24}$ T. Kwapień, U. Eichmann, and W. Sandner, Phys. Rev. A 75, 063418 (2007).

${ }^{25}$ H. Rohde, S. T. Gulde, C. F. Roos, P. A. Barton, D. Leibfried, J. Eschner, F. Schmidt-Kaler, and R. Blatt, J. Opt. B: Quantum Semiclassical Opt. 3, S34 (2001).

${ }^{26}$ P. O. Schmidt, T. Rosenband, C. Langer, W. M. Itano, J. C. Bergquist, and D. J. Wineland, Science 309, 749 (2005).

${ }^{27}$ D. B. Hume, C. W. Chou, D. R. Leibrandt, M. J. Thorpe, D. J. Wineland, and T. Rosenband, Phys. Rev. Lett. 107, 243902 (2011).

${ }^{28}$ H. G. Dehmelt, Bull. Am. Phys. Soc. 20, 60 (1975).

${ }^{29}$ J. C. Berengut, V. A. Dzuba, and V. V. Flambaum, Phys. Rev. Lett. 105, 120801 (2010)

${ }^{30}$ J. C. Berengut, V. A. Dzuba, V. V. Flambaum, and A. Ong, Phys. Rev. Lett. 106, 210802 (2011).
${ }^{31}$ S. W. Epp, J. R. C. López-Urrutia, G. Brenner, V. Mäckel, P. H. Mokler, R. Treusch, M. Kuhlmann, M. V. Yurkov, J. Feldhaus, J. R. Schneider, M. Wellhöfer, M. Martins, W. Wurth, and J. Ullrich, Phys. Rev. Lett. 98, 183001 (2007).

${ }^{32}$ J. R. Crespo López-Urrutia, Can. J. Phys. 86, 111 (2008).

${ }^{33}$ V. Mäckel, R. Klawitter, G. Brenner, J. R. Crespo López-Urrutia, and J. Ullrich, Phys. Rev. Lett. 107, 143002 (2011).

${ }^{34}$ S. Sturm, A. Wagner, B. Schabinger, J. Zatorski, Z. Harman, W. Quint, G. Werth, C. H. Keitel, and K. Blaum, Phys. Rev. Lett. 107, 023002 (2011).

${ }^{35}$ M. Zolotorev and D. Budker, Phys. Rev. Lett. 78, 4717 (1997).

${ }^{36}$ A. Surzhykov, A. Volotka, F. Fratini, J. P. Santos, P. Indelicato, G. Plunien, T. Stöhlker, and S. Fritzsche, Phys. Rev. A 81, 042510 (2010).

${ }^{37}$ V. M. Shabaev, A. V. Volotka, C. Kozhuharov, G. Plunien, and T. Stöhlker, Phys. Rev. A 81, 052102 (2010).

${ }^{38}$ S. Schiller, Phys. Rev. Lett. 98, 180801 (2007).

${ }^{39}$ P. Herskind, "Cavity quantum electrodynamics with ion Coulomb crystals," Ph.D. dissertation (Aarhus University, 2008).

${ }^{40}$ P. F. Herskind, A. Dantan, J. P. Marler, M. Albert, and M. Drewsen, Nat. Phys. 5, 494 (2009).

${ }^{41}$ A. Dantan, M. Albert, J. P. Marler, P. F. Herskind, and M. Drewsen, Phys. Rev. A 80, 041802 (2009).

${ }^{42}$ H. G. Barros, A. Stute, T. E. Northup, C. Russo, P. O. Schmidt, and R. Blatt, New J. Phys. 11, 103004 (2009).

${ }^{43}$ C. Russo, H. Barros, A. Stute, F. Dubin, E. Phillips, T. Monz, T. Northup, C. Becher, T. Salzburger, H. Ritsch, P. Schmidt, and R. Blatt, Appl. Phys. B: Lasers Opt. 95, 205 (2009).

${ }^{44}$ F. Dubin, C. Russo, H. G. Barros, A. Stute, C. Becher, P. O. Schmidt, and R. Blatt, Nat. Phys. 6, 350 (2010).

${ }^{45}$ A. Dantan, J. P. Marler, M. Albert, D. Guénot, and M. Drewsen, Phys. Rev. Lett. 105, 103001 (2010).

${ }^{46}$ P. F. Staanum, K. Højbjerre, P. S. Skyt, A. K. Hansen, and M. Drewsen, Nat. Phys. 6, 271 (2010)

${ }^{47}$ B. Schneider, T. Roth, H. Duncker, I. Ernsting, and S. Schiller, Nat. Phys. 6, 275 (2010).

${ }^{48}$ L. Gruber, J. P. Holder, J. Steiger, B. R. Beck, H. E. DeWitt, J. Glassman, J. W. McDonald, D. A. Church, and D. Schneider, Phys. Rev. Lett. 86, 636 (2001).

${ }^{49}$ M. Bussmann, U. Schramm, D. Habs, V. Kolhinen, and J. Szerypo, "Ultraaccurate mass spectrometry and related topics," Int. J. Mass Spectrom. 251, 179 (2006).

${ }^{50}$ A. Müller and E. Salzborn, Phys. Lett. A 62, 391 (1977).

${ }^{51}$ C. Benvenuti, J. Vac. Sci. Technol. 11, 591 (1974).

${ }^{52}$ M. E. Poitzsch, J. C. Bergquist, W. M. Itano, and D. J. Wineland, Rev. Sci. Instrum. 67, 129 (1996).

${ }^{53}$ K. Okada, M. Wada, T. Nakamura, I. Katayama, L. Boesten, and S. Ohtani, Jpn. J. Appl. Phys. 40, 4221 (2001).

${ }^{54}$ J. Labaziewicz, Y. Ge, P. Antohi, D. Leibrandt, K. R. Brown, and I. L. Chuang, Phys. Rev. Lett. 100, 013001 (2008).

${ }^{55}$ P. B. Antohi, D. Schuster, G. M. Akselrod, J. Labaziewicz, Y. Ge, Z. Lin, W. S. Bakr, and I. L. Chuang, Rev. Sci. Instrum. 80, 013103 (2009).

${ }^{56} \mathrm{M}$. Drewsen and A. Brøner, Phys. Rev. A 62, 045401 (2000).

${ }^{57}$ N. Kjærgaard and M. Drewsen, Phys. Rev. Lett. 91, 095002 (2003).

${ }^{58}$ N. Kjærgaard, K. Molhave, and M. Drewsen, Nucl. Instrum. Methods Phys. Res., Sect. A 540, 209 (2005).

${ }^{59}$ M. Drewsen, Eur. Phys. J. D 45, 125 (2007)

${ }^{60}$ P. Herskind, A. Dantan, M. Langkilde-Lauesen, A. Mortensen, J. Sørensen, and M. Drewsen, Appl. Phys. B: Lasers Opt. 93, 373 (2008).

${ }^{61}$ I. N. Laboratory, SIMION 8.0 User Manual.

${ }^{62} \mathrm{~T}$. Ballance, "Commissioning of a cryogenic Paul trap for highly charged ions," M.S. thesis (Imperial College London, 2011).

${ }^{63}$ N. Kjærgaard, L. Hornekaer, A. M. Thommesen, Z. Videsen, and M. Drewsen, Appl. Phys. B: Lasers Opt. 71, 207 (2000).

${ }^{64}$ T. W. Hänsch and B. Couillaud, Opt. Commun. 35, 441 (1980).

${ }^{65}$ R. W. P. Drever, J. L. Hall, F. V. Kowalski, J. Hough, G. M. Ford, A. J. Munley, and H. Ward, Appl. Phys. B: Lasers Opt. 31, 97 (1983).

${ }^{66}$ K. Mølhave and M. Drewsen, Phys. Rev. A 62, 011401 (2000).

${ }^{67}$ J. B. Wübbena, S. Amairi, O. Mandel, and P. O. Schmidt, Phys. Rev. A 85, 043412 (2012).

${ }^{68}$ A. C. Wilson, C. Ospelkaus, A. P. VanDevender, J. A. Mlynek, K. R. Brown, D. Leibfried, and D. J. Wineland, Appl. Phys. B: Lasers Opt. 105, 741 (2011). 